\title{
Infectious agents in the pathogenesis of primary biliary cirrhosis
}

\author{
Oscar-Danilo Ortega-Hernandez ${ }^{\mathrm{a}}$, Nancy-Agmon Levin ${ }^{\mathrm{a}, \mathrm{b}}$, Arie Altman $^{\mathrm{a}, \mathrm{b}}$ and Yehuda Shoenfeld ${ }^{\mathrm{a}, \mathrm{b}, *, 1}$ \\ ${ }^{a}$ The Zabludowicz Center for Autoimmune Diseases and Department of Medicine B, Sheba Medical Center, \\ Tel-Aviv, Israel \\ ${ }^{\mathrm{b}}$ Tel-Aviv University, Tel-Aviv, Israel
}

\begin{abstract}
Primary biliary cirrhosis (PBC) is a chronic progressive cholestatic liver disease which is characterized by the breakdown of self-tolerance to the highly conserved pyruvate dehydrogenase complex, specially the pyruvate dehydrogenase E2 complex (PDC-E2). The breakdown of the tolerance to such antigens leads to an autoimmune process characterized by portal inflammation and immune-mediated destruction of the intrahepatic bile ducts. Epidemiological studies have suggested that infections agents can trigger or even exacerbate the disease. Among other gram negative bacteria, Escherichia Coli, and Nosphingobium aromaticivorans are the most associated agents reported hitherto. Epidemiological and molecular evidence points towards molecular mimicry between some components of these microorganisms and specific amino-acid sequences that are present in proteins on normal cells of the biliary tract. In this review, we revisit all reports suggesting that infectious agents might be associated with the autoimmune pathogenesis of PBC. We also retrieve the immune molecular mimicry mechanisms that are likely involved with the autoimmune process in PBC.
\end{abstract}

Keywords: Primary biliary cirrhosis, infectious agents, molecular mimicry, tolerance, autoimmunity

\section{Introduction}

Primary biliary cirrhosis (PBC) is a chronic progressive cholestatic liver disease that affects mostly women over the age of 40 years and may progress to cirrhosis and liver failure [1]. The etiology of PBC is unknown, but considerable evidence points to a multi-causal process, involving an autoimmune activation triggered by environmental factors on the basis of a genetic background. Limited studies have indicated the association of HLA-DR8 with PBC [2], but in general, no particular HLA haplotype is considered to be associated with the occurrence of PBC. However genetic predisposition is strongly supported by almost 50 to 100 fold higher relative risk in first-degree relatives as well as the highest concordance rate between identical twins when com-

\footnotetext{
* Corresponding author: Prof. Yehuda Shoenfeld, Head, Department of Medicine B, Chaim Sheba Medical Center. Tel Hashomer 52621, Israel. Tel.: +972 3530 2652; Fax: +972 3535 2855; E-mail: shoenfel@post.tau.ac.il.

${ }^{1}$ Incumbent of the Laura Schwarz-Kip Chair for Research of Autoimmune Diseases.
}

pared to other autoimmune diseases (ADs) [3]. There are also some differences regarding gender, PBC predominates mainly in women with a ratio of 10 to 1 , suggesting some hormonal influence [1]. From a molecular perspective, $\mathrm{PBC}$ is characterized by the breakdown of self-tolerance to the highly conserved pyruvate dehydrogenase complex, specially the pyruvate dehydrogenase E2 complex (PDC-E2) [4]. Although, there is no general consensus, ten long amino acid row residues 163 through 176 carrying a lipoyl domain site in human PDC-E2 (163-176) are thought to be the prominent immunodominant epitope. Extensive studies have shown that that amino acid residues $\mathrm{E}, \mathrm{D}$, and $\mathrm{K}$ at positions 170,172 , and 173 , respectively, are essential for recognition by the T-cell clones [5,6]. Comparison in homology of some bacterial antigens and normal human PDC-E2 has shown high degree of identity in such critical amino-acid sequence and also antibodies against PDC-E2 (163-173) are able to cross-react with specific bacteria components. Likewise, epidemiological studies have suggested that infections can trigger or even exacerbate the disease [7,8]. In this regard, 
several infectious candidates have been suggested as potential causative agents of PBC [9-11], Escherichia Coli, Chlamydia Pneumoniae, Mycobacterium gordonae, and Nosphingobium aromaticivorans among others have been reported [7,12-15]. The evidence points towards molecular mimicry between some components of microorganisms and specific amino-acid sequences that are present in proteins on normal cells of the biliary tract $[4,7]$. Herein, we review all reports suggesting that infectious agents might be associated with the autoimmune pathogenesis of PBC.

\section{Autoimmune mechanisms in PBC and cross-reactivity}

PBC is the result of the breakdown of self tolerance to the mitochondrial and nuclear antigens as well as profound changes in the adaptive immunity $[4,16]$. The dominant antibody response in PBC is directed to the evolutionarily pyruvate 2-oxo-acid dehydrogenase complexes (2-OADC) located in the inner mitochondrial membrane. In this regard, the diagnostic hallmark of PBC is the presence of antimitochondrial antibodies (AMA) in $95 \%$ of the patients. The targets of the AMA includes the E2 subunits of the pyruvate dehydrogenase complex, the branched-chain 2-oxo-acid dehydrogenase complex, the ketoglutaric acid dehydrogenase complex, and the dihydrolipoamide dehydrogenase binding protein [17]. Although AMA are not the direct cause of biliary damage, they can predict disease onset and are detectable long before the clinical and histological features of PBC develop [18].

Most commonly, antibodies react against PDCE2 $[1,17]$. The E2 enzymes have a common structure consisting of the N-terminal domain containing the lipoyl group (Fig. 1). A number of studies using oligopeptides or recombinant proteins have demonstrated that the dominant epitope recognized by AMA is located within the lipoyl domain. Gershwin et al. [19]. suggested the important role of lipoic acid in determining the immunogenicity of mithocondrial antigens in $\mathrm{PBC}$, and also hypothesized that modifications of this domain are involved in the breakdown of tolerance in animal models [20]. Several studies have suggested that microbial infection has a role in the induction of AMA, through a mechanism of molecular mimicry [9, 14,21]. Protein data based studies have shown that more than 10 microbial sequences have high degree of similarity with human PDC-E2 [14]. Reactivity and competitive cross-reactivity sera studies between human mitochondrial and microbial components have shown competitive inhibition, suggesting a positive association $[9,14,21]$. The large number of immunogenic mimics may account for the dominance of the PDC-E2 in the pathogenesis of the disease.

Regarding $\mathrm{T}$ Cell response against mitochondrial antigens, the frequencies of CD8+ T cells, natural killer T-cells, and B-cells that are reactive with the PDCE2 are also higher in number in the liver than in peripheral blood [22]. Activated T-cells transferred into naive animals induce bile duct lesions resembling human PBC [23]. Furthermore, CD4+ autoreactive T cells have been identified even in AMA-negative PBC patients [19]. These data suggest that although AMA have a limited role in disease development, the whole antimitochondrial multi-lineage immune response address by autoantibodies, B cells, CD4+ and CD8+ T cells mainly directed to PDC-E2 might constitute the initial immunological insult in PBC [19,24].

\section{Infectious agents in PBC}

Several infectious candidates have been suggested as causative agents of PBC and molecular mimicry of human mitochondrial epitopes to microbial antigens is the most widely proposed mechanism [14]. Molecular mimicry is a direct mechanism, where the infection agents present an epitope structurally similar to a selfantigen [25]. As described above PDC-E2, the major antimitochondrial autoantigen, is a well-conserved sequence among various species, with a high degree of similarity to microbial PDC sequences of some microorganisms such as E. coli, Helicobacter pylori, cytomegalovirus among others [14]. In one study, AMA from patients with $\mathrm{PBC}$ were able to cross-react with PDC-E2 components derived from such microorganisms [26]. Although this theory is the most widely accepted, there is another potential mechanism known as epitope spreading, in which, infection accelerates an ongoing autoimmune process by the enhancement of local activation of antigen-presenting cells and overprocessing of antigens in individuals prone to develop PBC [27,28]. Even though infectious agents have been associated with $\mathrm{PBC}$, it is important to note that molecular similarity and cross-reactivity alone do not necessarily imply that the infectious agents can cause PBC by themselves, or that the initial immune response is directed to the microbial PDC-E2. It rather implies that this is a multi-causal process occurring in genetic prone patients [29]. 


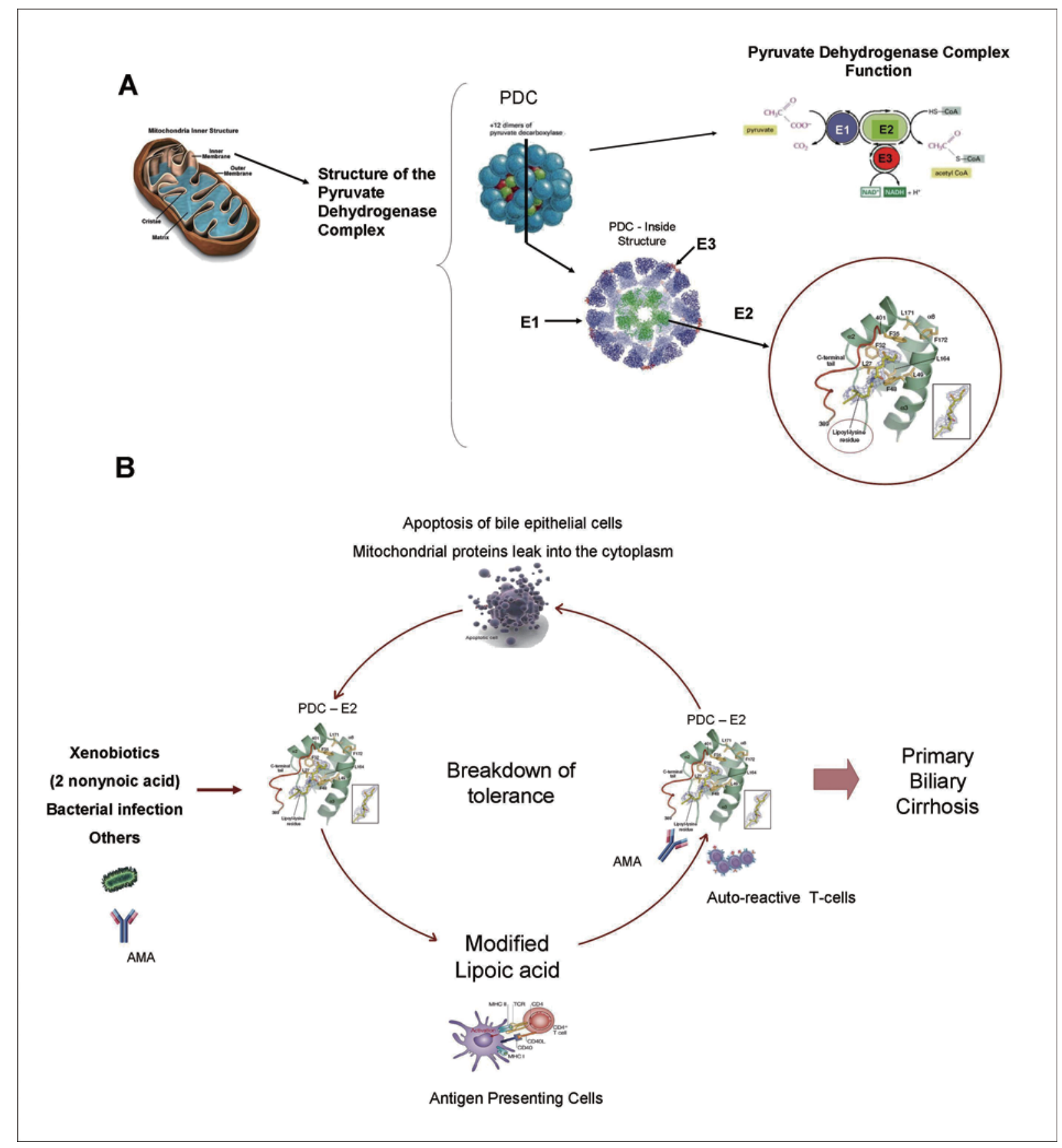

Fig. 1. (A) The structure of the pyruvate dehydrogenase a multienzyme complex (PDC), which is located on the inner membrane of mitochondria. It contains 60 polypeptide chains organized in three mean complexes 2-oxo acid dehydrogenase (E1 subunit), dihydrolipoamide acyltransferase (E2 subunit), and dihydrolipoamide dehydrogenase (E3 subunit). The three enzymes are coupled and reaction intermediates are passed directly from one enzyme to another. PDC converts pyruvate to acetyl CoA in the mitochondrial matrix; NADH is also produced in this reaction. The E2 subunits carry lipoic acid, an essential cofactor, which is covalently bound to the lysine residue within the lipoylated domain of the protein. (B) Apoptosis of bile epithelial cells might expose critical epitopes with high degree of homology in specific aminoacid sequences with antigens from several infectious agents. In addition to such process, environmental risk factors (xenobiotics), and other unknown factors may cause modification in the lipoic acid causing the break of tolerance and the onset of an autoimmune destructive process against mitochondrial normal components. Antimitochondrial antibodies (AMA) are mainly directed against the lipoyl domain site inside PDC-E2. AMA are able to cross react with both human and infectious agents antigens. 


\subsection{Nosphingobium aromaticivorans}

$N$. aromaticivorans is a gram negative strictly aerobic bacteria which is found worldwide in soil, water, and coastal plain sediments that metabolizes xenobiotics and activates environmental estrogens. $N$. aromaticivorans has been suggested as a pathogenic cofactor in the development of PBC [30]. In one study, it was reported that antibodies against $N$. aromaticivorans were found in $100 \%$ of 77 PBC Italian patients and cross-reacted with human PDC-E2 [31]. An important question was whether this finding was generalizable or only limited to PBC patients from Italy. To address this Olafsson et al. studied 14 Iceland patients with PBC, and 85 first-degree relatives [32]. The majority of Icelanders are of Scandinavian and Celtic origin and genetically distinct from Italian population. Thirteen sera samples of 14 Icelandic PBC patients who were positive for antibodies against $\mathrm{N}$. aromaticivorans reacted against at least one of the 2 oxo-acid dehydrogenase-E2 complexes [32]. It is of interest that one healthy first-degree relative had antibodies to $N$. aromaticivorans and developed PBC two years later. Approximately 25\% of PBC patients and controls had $N$. aromaticivorans in their stools but only those with PBC had antibodies to N. aromaticivorans. Earlier reports described an association between PBC and drinking water from one reservoir in Northeast England, raising the possibility of a common exposure to $N$. aromaticivorans from the water supply [33,34].

In support of this notion, studies in mice have shown that infection with $N$. aromaticivorans induced the appearance of antibodies against microbial PDC-E2 and its mitochondrial counterpart. In addition to the cell injury, concomitant production of anti-PDC-E2 antibodies that cross-react with conserved PDC-E2 epitopes display by $N$. aromaticivorans was also confirmed [35]. It also triggered chronic $\mathrm{T}$ cell-mediated autoimmunity against small bile ducts [35]. Furthermore, the infection leads to the development of liver lesions resembling human PBC. Of 13 contiguous amino acids comprising the core sequence of human PDC-E2 (GDLLAEIETDKAT), 12 are identical and only 1 is different in the corresponding sequence of N. aromaticivorans (Table 1). it has been shown that $N$. aromaticivorans exhibits PDC-E2-like proteins in their membranes with higher degree of homology to the immunodominant region of human PDC-E2 than any microorganism thus far studied (100-1,000 times greater than that of Escherichia coli) [36].
Furthermore, $N$. aromaticivorans can metabolize xenobiotics that are similar to the chemical compounds that react with sera from PBC patients [37]. Some of these xenobiotics are immunologically related to lipoic acid, the cofactor that is at the active center of PDCE2. Even more, Xenobiotic incorporation into PDC can occur [38]. Thus, N. aromaticivorans can theoretically break down self-tolerance in two ways: by molecular mimicry due to subclinical infection and by the metabolism of xenobiotics that are present in the environment $[37,38]$.

\subsection{Escherichia coli}

Molecular mimicry between E. coli and human PDCE2 was first described by Burroughs et al. [39]. Intestinal colonization by $\mathrm{R}$ (rough)-forms $E$. coli was found in the stool of 22 PBC patients (100\%) vs. 1 of 20 healthy controls as well as in $25 \%$ of patients with other liver diseases [40]. A high affinity of antibodies to PDC-E2 of $E$. coli was reported to be 100-fold higher in PBC patients [41]. Several studies have found an epidemiological association between $E$. coli and PBC [3,42]. The main finding comes from a controlled epidemiological analysis showing a positive association of PBC with recurrent urinary tract infection (UTI) [42]. Another epidemiological study performed on 1,032 PBC patients followed-up in 20 tertiary referral centers, and including 1,041 demographically-matched controls also confirmed the connection of UTI with PBC [3]. Reactivity to PDC-E2 up to $52 \%$ of sera from patients with chronic UTI was reported [43]. Other studies found that at least $33 \%$ of patients with PBC have antibodies that cross react to the ATP-dependent Clp protease of E. coli, especially to one specific aminoacid sequence (177-194) located inside the protein [44,45]. Moreover, the epitope of E. coli also maps to similar lipoyl domains which are present in human PDC-E2 [14] (Table 1). Such domain has been shown to be essential for T cell epitope recognition of both human PDC-E2 and E. coli PDC-E2 proteins $[14,46]$. This reactivity is strongly associated with a history of recurrent UTI [14]. Although there is a large amount of information supporting such an association, it is important to remark that antibodies to $E$. coli $\mathrm{PDC}$-E2 are more frequent in the later stages of the disease and in low titers, whereas antibodies to $N$. aromaticivorans are found 1000 times higher in early stages of the disease [31]. 
O.-D. Ortega-Hernandez et al. / Infectious agents in the pathogenesis of primary biliary cirrhosis

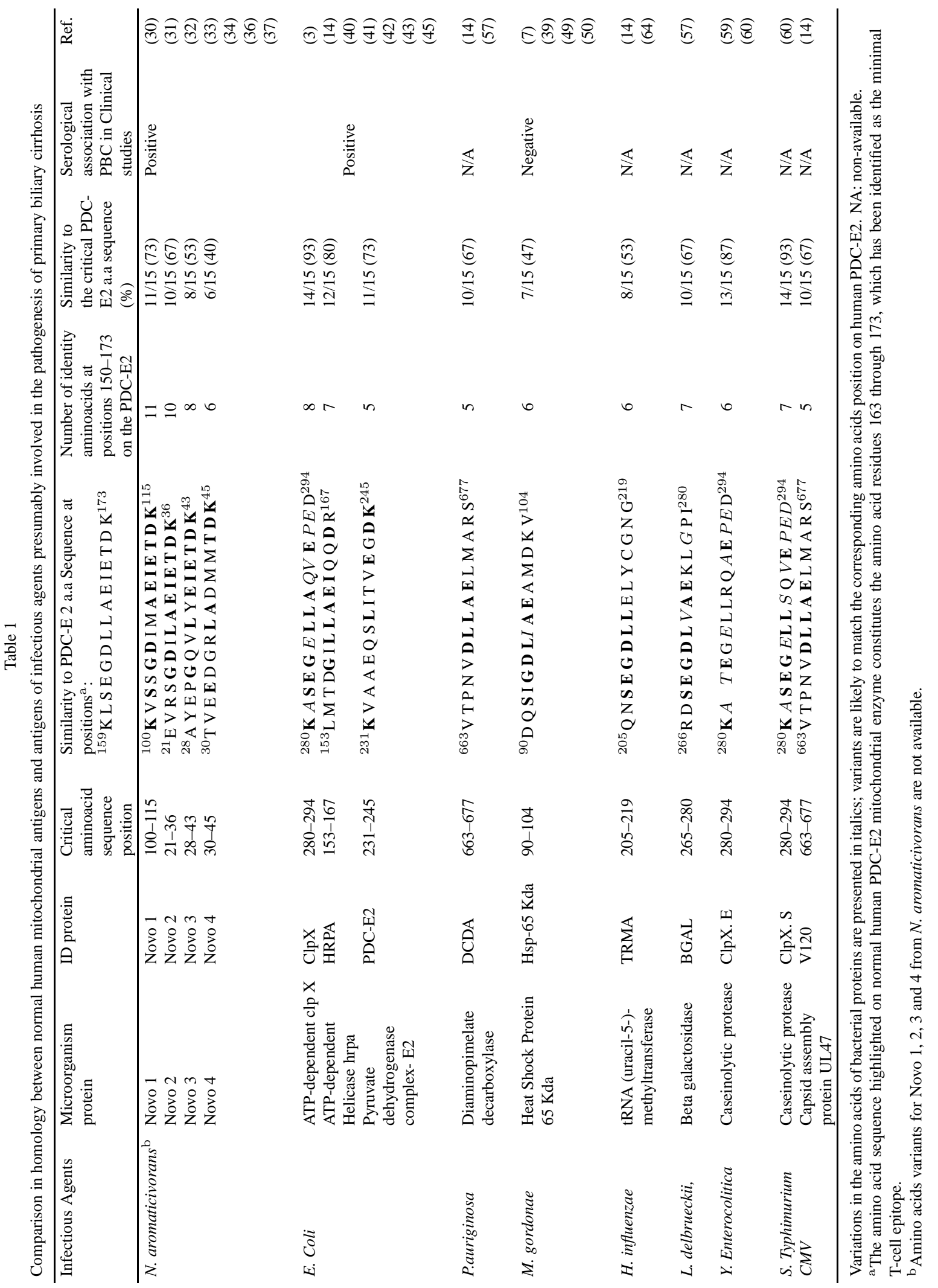




\subsection{Chlamydia pneumoniae}

A potential role for $C$. pneumoniae as a triggering agent of PBC was suggested in one study performed by Abdulkarim et al. [47]. From 39 patients with PBC who were enrolled, C. pneumoniae antigens were present in the $100 \%$ as compared to $8.5 \%$ of 105 controls biopsies including patients with primary sclerosing cholangitis and chronic hepatitis $\mathrm{C}$ virus infection. This association detected by immunohistochemistry and confirmed by in situ hybridization was specific for C. pneumoniae whereas any association was found with Chlamydia trachomatis which was also consistently negative in controls [47]. In another study, reactivity to Chlamydia antigens was found in the serum of 91\% AMA-positive and 21\% AMA-negative PBC patients but was practically absent in pathological and normal controls [48]. The presence of -16s-RNA from C. pneumoniae was demonstrated by transcription-polymerase chain reaction (PCR) in liver samples of those patients [48]. However, other authors have suggested that Chlamydia infection is not directly involved in PBC, rather, those findings might represent an epiphenomenon [13].

\subsection{Mycobacterium spp}

An association between infection with Mycobacteria and PBC has been seen since a long time ago. One early study reported that serum from patients with $\mathrm{PBC}$ was able to react to Mycobacterium spp [49]. Another study determined that binding can be done to one specific antigen, the head shock protein $-65 \mathrm{KDa}$ (hsp65) which is especially present in Mycobacterium gordonae. The sera of such patients did not react to other Mycobacterium species [7,50]. Burroughs et al. [39]. demonstrated a high degree of homology between hsp65 of Mycobacterium gordonae with human PDC-E2 (Table 1) [39]. In the same study, patients from two different geographical regions were included but surprisingly the sera of patients from one of the regions did not react to the hsp65 of Mycobacterium gordonae. The authors remark that atypical bacteria (Mycobacterium gordonae) are less prevalent in that region which can be responsible of the absence of serological reaction [39]. Such findings are also in agreement with disparities found regarding the incidence of PBC among different populations [51,52]. In general, differences in infectious diseases and the incidence of ADs are found from one country to another [53, 54]. ADs are not evenly distributed among continents, well-circumscribed regions within a given country, or ethnic groups. An examination of the distribution reveals several important and probably interrelated phenomena. One is the north-south gradient: the incidence of disease decreases from north to south in the Northern Hemisphere (and reciprocally from south to north in the Southern Hemisphere). Epidemiologic data provide strong evidence of a steady rise in the incidence of ADs in developed countries over the past three decades. Concomitantly, there has been an obvious decrease in the incidence of many infectious diseases in developed countries as a result of antibiotics, vaccination, or more simply, improved hygiene and better socioeconomic conditions [55]. A plausible explanation for such findings might be the limited exposure to a significant infectious agent's burden in the North-South gradient. Another example of this gradient regarding $\mathrm{PBC}$ is the presence of AMA in patients with active pulmonary tuberculosis and active leprosy in almost $43 \%$ of patients. However none of them developed $\mathrm{PBC}$, suggesting that at least the exposure to a single agent per se is not critical in the development of the disease [26,56]. Furthermore, Mycobacterium species have not been isolated from any histological samples of patients with PBC. Attempts to detect mycobacterial DNA in the histological samples has also failed [49]. Therefore, the role of Mycobacterium gordonae in the pathogenesis of PBC remains uncertain.

\subsection{Lactobacillus spp}

The group of Bogdanos et al. [57]. described the presence of autoantibodies to human PDC-E2 able to cross-react with a Lactobacillus-mimic antigen. The authors reported that the enzyme beta galactosidase (lactase) of L. delbrueckii, sub-specie bulgaricus shares eight aminoacid sequences with human PDC-E2 (213226) (Table 1). Such association seems to be the highest when compared to other Lactobacillus species [57]. Although Lactobacillus does not cause infection, the enzyme lactase of Lactobacillus is broadly used in the production of lacteous for human consumption. It was suggested that fermented products might constitute another environmental risk factor for PBC. Regarding cross-reactivity, the same group also showed that the serum of more than $50 \%$ of PBC patients with positive AMA -subclass IgG3 are able to react with lactase of $L$. delbrueckii, with high specificity [57]. The affinity of the antibody for $L$. delbrueckii mimic was greater than for human PDC-E2. 


\subsection{Other gram negative bacteria and $P B C$}

Apart from E. Coli, other gram negative bacteria have been reported to be epidemiologically associated with PBC prevalence. The common antigenic denominator for such gram negative bacteria is the presence of the ATP-dependent caseinolytic protease (Clp). ClpP is a serine protease complex that together with the regulatory subunit ClpX plays an important physiological role in protein degradation [58]. The Clp protease complex is broadly distributed among gram negative bacteria, and its structure is highly conserved. ClpP of E. coli is identical to the corresponding ClpP of Salmonella typhimurium and also has a high degree of homology with ClpP of Yersinia enterocolitica [in particular to one amino acid sequence of $\mathrm{Clp}(177-194)]$, and to a lesser extent to ClpP of Haemophilus influenza [14,58] (Table 1). Mayo et al. [45]. enrolled 45 patients with PBC, 44 patients with Hepatitis $\mathrm{C}$ and autoimmune thyroiditis (AT) and 32 healthy controls. Reactivity to at least one of the ClpP peptides was observed in 21 (47\%) of PBC patients, $5.8 \%$ of patients with Hepatitis $\mathrm{C}$ and $\mathrm{AT}$ and $3.1 \%$ of healthy controls. Among these 21 seropositive $\mathrm{PBC}$ patients, $71 \%$ reacted at least to the amino acid sequence 177-194, located within the proteolytic subunit of the Clp E. Coli, alone or in association with Clp of $Y$. Enterocolitica and/or Clp of $H$. Influenzae peptides [45]. Simultaneous reactivity to homologous sequences was also observed. By competition assays, the authors confirmed that it was due to cross-reactivity [45]. In a large follow-up study of patients with infection by $Y$. Enterocolitica, the induction of chronic liver diseases and stimulation of various non-organ specific autoantibodies was observed in some patients [59]. Although previous reports suggest cross reactivity, the overall frequency of infection by $Y$. Enterocolitica has been described to be quite similar between PBC and non-PBC controls [60]. No additional epidemiological studies searching for such association have been performed.

\subsection{Viral infectious agents}

Although the association between virus infection and $\mathrm{PBC}$ is less clear compared with bacteria, there are some remarkable studies supporting such association. Two herpes virus have been described to share sequence homology with human PDC-E2. The first one is Epstein-Barr virus (EBV), a virus belonging to the herpes family, which infection has been largely associated with multiple ADs $[61,62]$. Recently, we have found in- creased titers of the early antigen of EBV in sera of patients with different ADs [63]. Morshed et al. [64]. had reported long time ago, that peripheral blood mononuclear cells of patients with PBC showed increased levels of EBV-DNA $(61 \%)$ in contrast to chronic active hepatitis patients (19\%), liver cirrhosis patients (14\%) and healthy individuals (11\%) [64]. They also showed that formalin-fixed paraffin-embedded liver tissues, and saliva from the same PBC patients, also demonstrated increased levels of EBV-DNA when compared to healthy individuals [64]. The second herpes virus is the cytomegalovirus (CMV) and even though no clinical studies have reported a direct association with PBC, one peptide sequence shows sharing similarity to the human PDC [14] (Table 1). Regarding other viruses, a plausible role for retroviruses was suggested by Mason et al. [65]. They reported a long time ago that the sera from patients with $\mathrm{PBC}$ react with proteins from the human immunodeficiency virus 1 (HIV1) and human intracisternal A-type particle (HIAP). The same group identified a human betaretrovirus that shares high homology with the mouse mammary tumor virus (MMTV) [66]. Then, MMTV was identified and cloned in the liver and lymph nodes of patients with PBC by the same group [66]. By means of PCR, it was only found in $20 \%$ of the serum samples that contained antiretroviral reactivity. This retrovirus appears to be more lymphotropic than hepatotropic $[65,66]$. It was also reported that co-culture of healthy biliary epithelial cells together with lymph node tissue from patients with PBC, lead to the expression of an AMA - specific auto-antigen on the apical surface of such epithelial cells $[67,68]$. Ultrastructure studies also suggested the presence of a viral-like antigen in PBC samples [69]. Otherwise, the group of Gershwin did not detect reactivity of the sera of patients with PBC against any MMTV encoded protein [70]. In the same study, two proteins of different molecular weight present in the MMTV extracts were recognized by a small proportion of PBC-sera with positive AMA. They demonstrated that such proteins were lipoylated and not recognized by any of the anti-MMTV polyclonal sera tested [70]. They also did not find either evidence of MMTV in liver samples obtained from patients with $\mathrm{PBC}$, nor specific sequences of MMTV in peripheral blood mononuclear cells of the same patients [70]. Therefore whether or not retroviruses are associated with the pathogenesis of PBC remains controversial. 


\section{Concluding remarks}

There is a large amount of evidence suggesting an association between infectious agents and PBC. Most of the evidence points to molecular mimicry between specific bacteria components and human mitochondrial antigens $[4,14,26]$. Although in vitro studies and some animal models support the concept of molecular mimicry in PBC [20,35], only some clinical studies support such association $[3,32,42]$. Some other studies have failed to find a direct relation [70-72]. Direct epidemiological association between exposure to a determined infectious agent and PBC onset remains unclear. In this regard, a plausible explanation for epidemiological differences in the incidence of PBC might be the variation in the exposure to several infectious agents burden rather than to a single agent exposure [54,55]. Clearly, whatever is the reason for this marked epidemiology discrepancy; we must admit that genetics and environment are both also involved. Therefore, it is worthy to remark that the association of PBC with infectious agents constitutes only one piece belonging to the "big puzzle", but the exact mechanism of disease's origin remains unknown. Other components such as genetic, hormonal, environmental, and xenobiotic also make PBC etiology enigmatic [73-76]. Moreover, the exact mechanism implicated in the induction of PBCspecific anti-mitochondrial responses is not clearly understood and since PBC is an autoimmune disease in which several aspects contribute to its pathogenesis, the interaction among them is also unknown [77]. Such interactions are highly amenable to additional exploration through new clinical and experimental studies.

\section{Acknowledgements}

O.D. Ortega and Y. Shoenfeld are recipients of Federico Foundation Wilhelm Agricola research grants.

\section{References}

[1] M.M. Kaplan and M.E. Gershwin, Primary biliary cirrhosis, N Engl J Med 353 (2005), 1261-1273.

[2] J. Underhill, P. Donaldson, G. Bray, D. Doherty, B. Portmann and R. Williams, Susceptibility to primary biliary cirrhosis is associated with the HLA-DR8-DQB1*0402 haplotype, Hepatology 16 (1992), 1404-1408.

[3] M.E. Gershwin, C. Selmi, H.J. Worman, E.B. Gold, M. Watnik, J. Utts et al., Risk factors and comorbidities in primary biliary cirrhosis: a controlled interview-based study of 1032 patients, Hepatology 42 (2005), 1194-1202.
[4] X.S. He, A.A. Ansari, W.M. Ridgway, R.L. Coppel and M.E. Gershwin, New insights to the immunopathology of autoimmune responses in primary biliary cirrhosis, Cell Immunol 239 (2006), 1-13.

[5] S. Braun, C. Berg, S. Buck, M. Gregor and R. Klein, Catalytic domain of PDC-E2 contains epitopes recognized by antimitochondrial antibodies in primary biliary cirrhosis, World $J$ Gastroenterol 16 (2010), 973-981.

[6] S.J. Yeaman, S.P. Fussey, D.J. Danner, O.F. James, D.J. Mutimer and M.F. Bassendine, Primary biliary cirrhosis: identification of two major M2 mitochondrial autoantigens, Lancet 1 (1988), 1067-1070.

[7] L. Vilagut, A. Parés, O. Viñas, J. Vila, M.T. Jiménez de Anta and J. Rodés, Antibodies to mycobacterial $65 \mathrm{kD}$ heat shock protein cross-react with the main mitochondrial antigens in patients with primary biliary cirrhosis, Eur J Clin Invest 27 (1997), 667-672.

[8] C. Selmi and M.E. Gershwin, Apocalypsal versus apocryphal: the role of retroviruses in primary biliary cirrhosis, Am J Gastroenterol 99 (2004), 2356-2358.

[9] C. Selmi and M.E. Gershwin, Bacteria and human autoimmunity: the case of primary biliary cirrhosis, Curr Opin Rheumatol 16 (2004), 406-410.

[10] N. Agmon-Levin, B.S. Katz and Y. Shoenfeld, Infection and primary biliary cirrhosis, Isr Med Assoc J11 (2009), 112-115.

[11] D.P. Bogdanos and D. Vergani, Bacteria and primary biliary cirrhosis, Clin Rev Allergy Immunol 36 (2009), 30-39.

[12] A.K. Burroughs, I.J. Rosenstein, O. Epstein, J.M. HamiltonMiller, W. Brumfitt and S. Sherlock, Bacteriuria and primary biliary cirrhosis, Gut 25 (1984),133-137.

[13] D. Taylor-Robinson, A.W. Sharif, N.S. Dahanjal and S.D. Taylor-Robinson, Chlamydia pneumoniae infection is an unlikely cause of primary biliary cirrhosis, J Hepatol 42 (2005), 778-780.

[14] D.P. Bogdanos, H. Baum, A. Grasso, M. Okamoto, P. Butler, Y. Ma et al., Microbial mimics are major targets of crossreactivity with human pyruvate dehydrogenase in primary biliary cirrhosis, J Hepatol 40 (2004), 31-39.

[15] J.P. Mohammed and J. Mattner, Autoimmune disease triggered by infection with alphaproteobacteria, Expert Rev Clin Immunol 5 (2009), 369-379.

[16] V. Barak, C. Selmi, M. Schlesinger, M. Blank, N. AgmonLevin, I. Kalickman et al., Serum inflammatory cytokines, complement components, and soluble interleukin 2 receptor in primary biliary cirrhosis, J Autoimmun 33 (2009), 178-182.

[17] M.E. Gershwin, A.A. Ansari, I.R. Mackay, Y. Nakanuma, A. Nishio, M.J. Rowley et al., Primary biliary cirrhosis: an orchestrated immune response against epithelial cells, Immunol Rev 174 (2000), 210-225.

[18] J.V. Metcalf, H.C. Mitchison, J.M. Palmar, D.E. Jones, M.E. Basssendine and O.F. James, Natural history of early primary biliary cirrhosis, Lancet 348 (1996), 1399-1402.

[19] M.E. Gershwin and I.R. Mackay, The causes of primary biliary cirrhosis:convenient and inconvenient truths, Hepatology 47 (2008), 737-745.

[20] Y. Ueno, Y.M. Ambrosini, Y. Moritoki, W.M. Ridgway and M.E. Gershwin, Murine models of autoimmune cholangitis, Curr Opin Gastroenterol 26 (2010), 274-479.

[21] J. Van de Water, H. Ishibashi, R.L. Coppel and M.E. Gershwin, Molecular mimicry and primary biliary cirrhosis: premises not promises, Hepatology 33 (2001), 771-775.

[22] H. Kita, S. Matsumura, X.S. He, A.A. Ansari, Z.X. Lian, J. Van de Water et al., Quantitative and functional analysis of PDC- 
E2 specific autoreactive cytotoxic T lymphocytes in primary biliary cirrhosis, J Clin Invest 109 (2002), 1231-1240.

[23] D.E.J. Jones, A. Robe, J. Palmer and J.A. Kirby, Adoptive transfer of self-PDC-reactive T-cells into naïve mice induces portal tract and bile duct changes characteristic of primary biliary cirrhosis (PBC), J Hepatol 44(Suppl 2) (2006), S10.

[24] H. Tanimoto, S. Shimoda, M. Nakamura, H. Ishibashi, A. Kawano, T. Kamihira et al., Promiscuous T cells selected by Escherichia coli: OGDC-E2 in primary biliary cirrhosis, $J$ Autoimmun 20 (2003), 255-263.

[25] N. Agmon-Levin, M. Blank, Z. Paz and Y. Shoenfeld, Molecular mimicry in systemic lupus erythematosus, Lupus 18 (2009), 1181-1185.

[26] R. Klein, M. Wiebel, S. Engelhart and P.A. Berg, Sera from patients with tuberculosis recognize the M2a-epitope (E2 subunit of pyruvate dehydrogenase complex) specific for primary biliary cirrhosis, Clin Exp Immunol 92 (1993), 308-316.

[27] P.V. Lehmann, T. Forsthuber, A. Miller and E.E. Sercarz, Spreading of T-cell autoimmunity for cryptic determinants of an autoantigen, Nature 358 (1992), 155-157.

[28] P.A. Berg, Autoimmunity in primary biliary cirrhosis: An alternative view at initiation and function of anti-mitochondrial autoantibodies, Hepatol 50 (2009), 827-828.

[29] Y. Ichiki, S. Shimoda, H. Ishibashi and M.E. Gershwin, Is primary biliary cirrhosis a model autoimmune disease, Autoimmun Rev 3 (2004), 331-336.

[30] M.M. Kaplan, Novosphingobium aromaticivorans: a potential initiator of primary biliary cirrhosis, Am J Gastroenterol 99 (2004), 2147-2149.

[31] C. Selmi, D.L. Balkwill, P. Invernizzi, A.A. Ansari, R.L. Coppel, M. Podda et al., Patients with primary biliary cirrhosis react against a ubiquitous xenobiotic-metabolizing bacterium, Hepatology 38 (2003), 1250-1257.

[32] S. Olafsson, H. Gudjonsson, C. Selmic, K. Amano, P. Invernizzi, M. Podda et al., Antimitochondrial antibodies and reactivity to $\mathrm{N}$. aromaticivorans proteins in Icelandic patients with primary biliary cirrhosis and their relatives, Am J Gastroenterol 99 (2004), 2143-2146.

[33] D.R. Triger, Primary biliary cirrhosis: An epidemiological study, Br Med J 281 (1980), 772-775.

[34] M.I. Pince, A. Chetwynd, P. Diggle, M. Jarner, J.V. Metcalf and O.F. James, The geographical distribution of primary biliary cirrhosis in well-defined cohort, Hepatology 34 (2001), 1083-1088.

[35] J. Mattner, P.B. Savage, P. Leung, S.S. Oertelt, V. Wang, O. Trivedi et al., Liver autoimmunity triggered by microbial activation of natural killer T cells, Cell Host Microbe 3 (2008), 304-315.

[36] K.A. Padgett, C. Selmi, T.P. Kenny, P.S. Leung, D.L. Balkwill, A.A. Ansari, R.L. Coppel et al., Phylogenetic and immunological definition of four lipoylated proteins from Novosphingobium aromaticivorans, implications for primary biliary cirrhosis, J Autoimmun 24 (2005), 209-219.

[37] T. Shi, J.K. Fredrickson and D.L. Balkwill, Biodegradation of polycyclic aromatic hydrocarbons by Sphingomonas strains isolated from the terrestrial subsurface, J Ind Microbiol Biotechnol 26 (2001), 283-289.

[38] H.R. Walden, J.A. Kirby, S.J. Yeaman, J. Gray, D.E. Jones and J.M. Palmer, Xenobiotic incorporation into pyruvate dehydrogenase complex can occur via the exogenous lipoylation pathway, Hepatology 48 (2008), 1874-1884.

[39] A.K. Burroughs, P. Butler, M.J. Sternberg and H. Baum, Molecular mimicry in liver disease, Nature 358 (1992), 377378.
[40] U. Hopf, B. Moller, R. Stemerowicz, H. Lobeck, A. Rodloff, M. Freudenberg et al., Relation between Escherichia coli R (rough)-forms in gut, lipid A in liver, and primary biliary cirrhosis, Lancet 2 (1989), 1419-1422.

[41] H. Miyakawa, A. Tanaka, C. Selmi, N. Hosoya, N. Mataki, K. Kikuchi et al., Serum reactivity against bacterial pyruvate dehydrogenase: increasing the specificity of anti mitochondrial antibodies for the diagnosis of primary biliary cirrhosis, Clin Dev Immunol 13 (2006), 289-294.

[42] A. Parikh-Patel, E.B. Gold, H. Worman, K.E. Krivy and M.E. Gershwin, Risk factors for primary biliary cirrhosis in a cohort of patients from the united states, Hepatology 33 (2001), 1621.

[43] P. Butler, J. Hamilton-Miller, H. Baum and A.K. Burroughs, Detection of M2 antibodies in patients with recurrent urinary tract infection using an ELISA and purified PBC specific antigens. Evidence for a molecular mimicry mechanism in the pathogenesis of primary biliary cirrhosis?, Biochem Mol Biol Int 35 (1995), 473-485.

[44] H. Baum, D.P. Bogdanos and D. Vergani, Antibodies to Clp protease in primary biliary cirrhosis: possible role of a mimicking T-cell epitope, J Hepatol 34 (2001), 785-787.

[45] I. Mayo, P. Arizti, A. Pares, J. Oliva, R.A. Doforno, M.R. de Sagarra et al., Antibodies against the $\mathrm{COOH}$-terminal region of E. coli ClpP protease in patients with primary biliary cirrhosis, J Hepatol 33 (2000), 528-536.

[46] S. Shimoda, M. Nakamura, H. Shigematsu, H. Tanimoto, T. Gushima, M.E. Gershwin et al., Mimicry peptides of human PDC-E2 163-176 peptide, the immunodominant T-cell epitope of primary biliary cirrhosis, Hepatology 31 (2000), 12121216.

[47] A.S. Abdulkarim, L.M. Petrovic, W.R. Kim, P. Angulo, R.V. Lloyd and K.D. Lindor, Primary biliary cirrhosis: an infectious disease caused by Chlamydia pneumoniae? J Hepatol 40 (2004), 380-384.

[48] P.S. Leung, O. Park, S. Matsumura, A.A. Ansari, R.L. Coppel and M.E. Gershwin, Is there a relation between Chlamydia infection and primary biliary cirrhosis? Clin Dev Immunol 10 (2003), 227-233.

[49] J. O'Donohue, B. McFarlane, A. Bomford, M. Yates and R. Williams, Antibodies to atypical mycobacteria in primary biliary cirrhosis, J Hepatol 21 (1994), 887-889.

[50] L. Vilagut, J. Vila, O. Vinas, A. Pares, A. Gines, M.T. Jimenez de Anta et al., Cross-reactivity of anti-Mycobacterium gordonae antibodies with the major mitochondrial autoantigens in primary biliary cirrhosis, J Hepatol 21 (1994), 673-677.

[51] S. Abu-Mouch, C. Selmi, G.D. Benson, T.P. Kenny, P. Invernizzi, M. Zuin et al., Geographic clusters of primary biliary cirrhosis, Clin Dev Immunol 10 (2003), 127-131.

[52] P. Invernizzi, Geoepidemiology of autoimmune liver diseases, J Autoimmun 34 (2010), 300-306.

[53] A.T. Borchers, S.M. Naguwa, Y. Shoenfeld and M.E. Gershwin, The geoepidemiology of systemic lupus erythematosus, Autoimmun Rev 9 (2010), 277-287.

[54] Y. Shapira, N. Agmon-Levin and Y. Shoenfeld, Defining and analyzing geoepidemiology and human autoimmunity, $\mathrm{J} A u-$ toimmun 34 (2010), 168-177.

[55] P. Youinou, J.O. Pers, M.E. Gershwin and Y. Shoenfeld, Geoepidemiology and autoimmunity, J Autoimmun 34 (2010), $163-167$.

[56] B. Gilburd, L. Ziporen, D. Zharhary, M. Blank, N. Zurgil, M.A. Scheinberg et al., Antimitochondrial (pyruvate dehydrogenase) antibodies in leprosy, J Clin Immunol 14 (1994), 14-19. 
[57] D.P. Bogdanos, H. Baum, M. Okamoto, P. Montalto, U.C. Sharma, E.I. Rigopoulou et al., Primary biliary cirrhosis is characterized by IgG3 antibodies cross-reactive with the major mitochondrial autoepitope and its Lactobacillus mimic, Hepatology 42 (2005), 458-465.

[58] S.K. Singh, R. Grimaud, J.R. Hoskins, S. Wickner and M.R. Maurizi, Unfolding and internalization of proteins by the ATPdependent proteases ClpXP and ClpAP, Proc Natl Acad Sci USA 97 (2000), 8898-8903.

[59] A. Saebo and J. Lassen, Acute and chronic liver disease associated with Yersinia enterocolitica infection: a Norwegian 10-year follow-up study of 458 hospitalized patients, J Intern Med 231 (1992), 531-535.

[60] D.P. Bogdanos, H. Baum, U.C. Sharma, A. Grasso, Y. Ma, A.K. Burroughs et al., Antibodies against homologous microbial caseinolytic proteases $\mathrm{P}$ characterise primary biliary cirrhosis, J Hepatol 36 (2002), 14-21.

[61] G.N. DeLorenze, K.L. Munger, E.T. Lennette, N. Orentreich, J.H. Vogelman and A. Ascherio, Epstein-Barr virus and multiple sclerosis: evidence of association from a prospective study with long-term follow-up, Arch Neurol 63 (2006), 839-844.

[62] E. Toussirot and J. Roudier, Epstein-Barr virus in autoimmune diseases, Best Pract Res Clin Rheumatol 22 (2008), 883-896.

[63] O. Barzilai, Y. Sherer, M. Ram, D. Izhaky, J.M. Anaya and Y. Shoenfeld, Epstein-Barr virus and cytomegalovirus in autoimmune diseases: are they truly notorious? A preliminary report, Ann N Y Acad Sci 1108 (2007), 567-577.

[64] S.A. Morshed, M. Nishioka, I. Saito, K. Komivama and I. Moro, Increased expression of Epstein-Barr virus in primary biliary cirrhosis patients, Gastroenterol Jpn 27 (1992), 751758.

[65] A.L. Mason, L. Xu, L. Guo, S. Munoz, J.B. Jaspan, M. BryerAsh et al., Detection of retroviral antibodies in primary biliary cirrhosis and other idiopathic biliary disorders, Lancet $\mathbf{3 5 1}$ (1998), 1620-1624.

[66] L. Xu, M. Sakalian, Z. Shen, G. Loss, J. Neuberger, A. Mason et al., Cloning the human betaretrovirus proviral genome from patients with primary biliary cirrhosis, Hepatology 39 (2004), $151-156$.

[67] T. Sadamoto, R. Joplin, A. Keogh, A. Mason, W. Carman and
J. Neuberger, Expression of pyruvate-dehydrogenase complex PDC-E2 on biliary epithelial cells induced by lymph nodes from primary biliary cirrhosis, Lancet 352 (1998), 1595-1596.

[68] A. Mason, L. Xu, Z. Shen, B. Fodera, R. Joplin, J. Neuberger et al., Patients with primary biliary cirrhosis make anti-viral and anti-mitochondrial antibodies to mouse mammary tumor virus, Gastroenterology 127 (2004), 1863-1864.

[69] L. Xu, Z. Shen, L. Guo, B. Fodera, A. Keogh, R. Joplin et al., Does a betaretrovius infection trigger primary biliary cirrhosis? Proc Natl Acad Sci USA 100 (2003), 8454-8459.

[70] C. Selmi, S.R. Ross, A.A. Ansari, P. Ivernizzi, M. Podda, R.L. Coppel et al., Lack of immunological or molecular evidence for a role of mouse mammary tumor retrovirus in primary biliary cirrhosis, Gastroenterology 127 (2004), 493-501.

[71] M. Durazzo, F. Rosina, A. Premoli, E. Morello, S. Fagoonee, R. Innarella et al., Lack of association between seroprevalence of Helicobacter pylori infection and primary biliary cirrhosis, World J Gastroenterol 10 (2004), 3179-3181.

[72] S.Y. Boomkens, S. de Rave, R.G. Pot, H.F. Egberink, L.C. Penning, J. Rothuizen et al., The role of Helicobacter spp. In the pathogenesis of primary biliary cirrhosis and primary sclerosing cholangitis, FEMS Immunol Med Microbiol 44 (2005), 221-225.

[73] B.D. Juran, E.J. Atkinson, J.J. Larson, E.M. Schlicht and K.N. Lazaridis, Common genetic variation and haplotypes of the anion exchanger SLC4A2 in primary biliary cirrhosis, Am J Gastroenterol 104 (2009), 1406-1411.

[74] C. Selmi and M.E. Gershwin, The role of environmental factors in primary biliary cirrhosis, Trends Immunol 30 (2009), 415-420.

[75] M.I. Pince, S.J. Ducker and O.F. James, Case-control studies of risk factors for primary biliary cirrhosis in two United Kingdom populations, Gut 59 (2010), 508-512.

[76] R. Rieger and M.E. Gershwin, The $X$ and why of xenobiotics in primary biliary cirrhosis, J Autoimmun 28 (2007), 76-84.

[77] R.J. McNally, S. Ducker and O.F. James, Are transient environmental agents involved in the cause of primary biliary cirrhosis? Evidence from space-time clustering analysis, Нерatology 50 (2009), 1169-1174. 


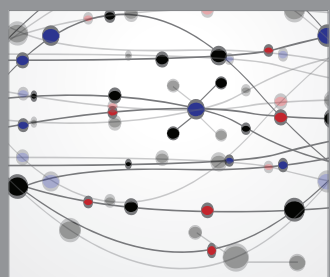

The Scientific World Journal
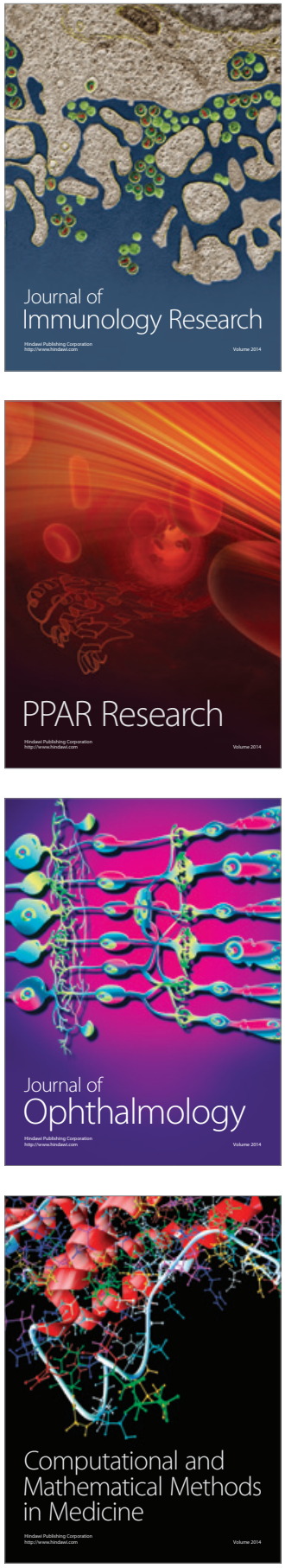

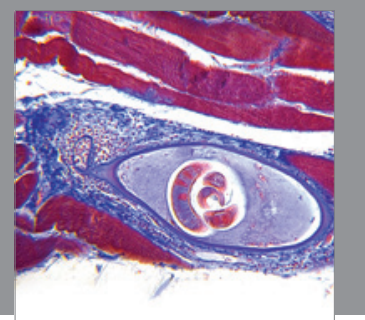

Gastroenterology

Research and Practice
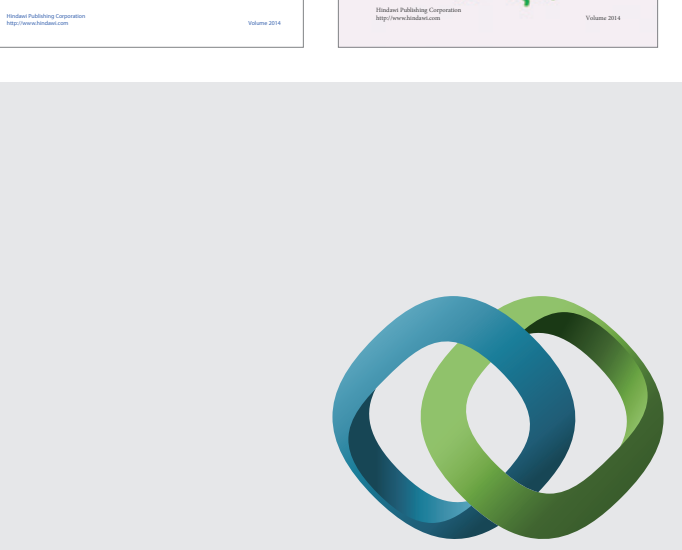

\section{Hindawi}

Submit your manuscripts at

http://www.hindawi.com
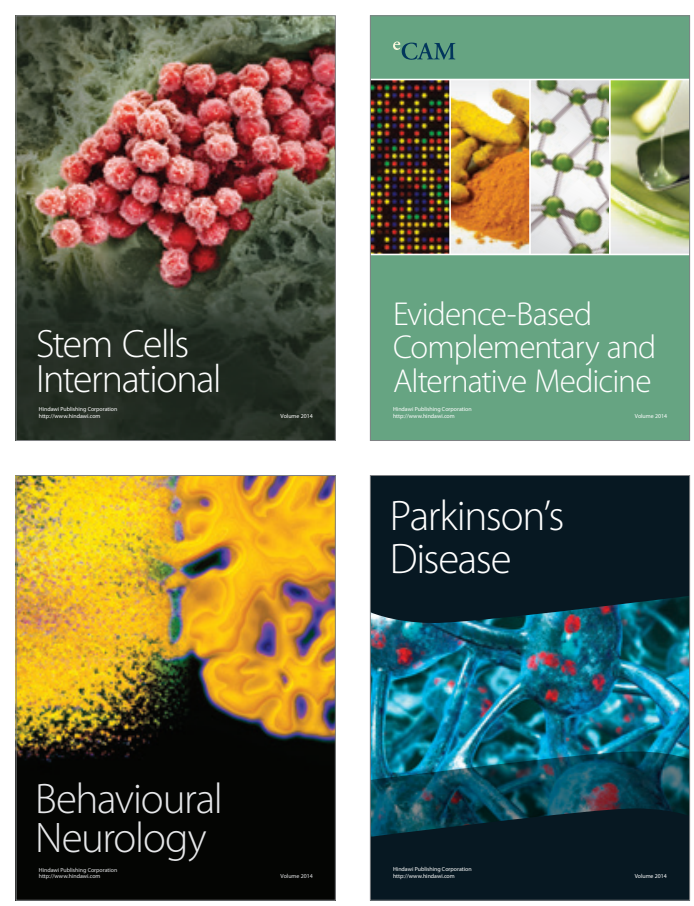

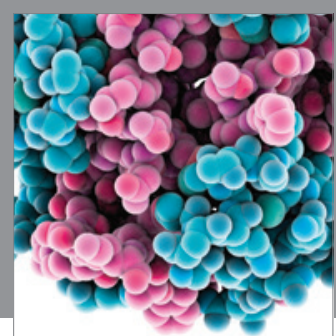

Journal of
Diabetes Research

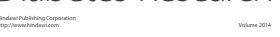

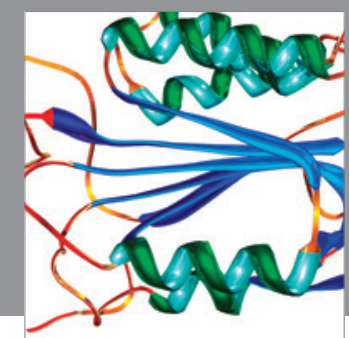

Disease Markers
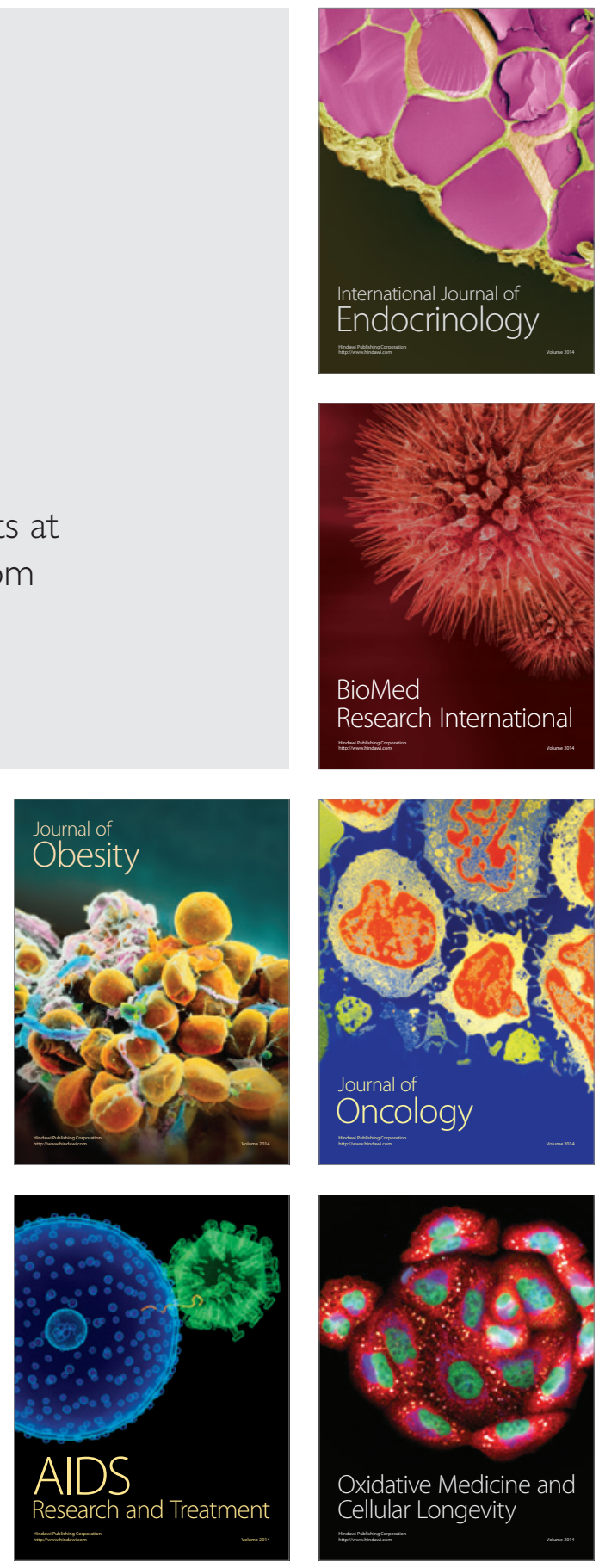